\title{
Fragen aus der Praxis
}

\section{Frage}

Die Theorie, nach der Geburt des kindlichen Kopfes auf die nächste Wehe zu warten, um den Rest des Kindes zu gebären, stützt sich auf die Auffassung, dass das Kind weiterhin über die Nabelschnur mit Sauerstoff versorgt wird, denn der Bauch des Kindes ist noch über dem Becken und somit keinem Druck ausgesetzt. Außerdem kann das Kind im Bedarfsfall atmen, da die Nase und der Mund ja geboren und frei sind. Weshalb ist dann bei einer verzögerten Schulterentwicklung, wie es bei der Schulterdystokie der Fall ist, das Überleben des Kindes gefährdet?'

Rose Kizler, Nouakchott, Mauretanien

\section{Antwort}

Die Frage, wie viel Zeit zwischen Kopfgeburt und Schulterentwicklung vergehen kann, ohne dass das Neugeborene geschädigt wird, ist schwer zu beantworten und bedarf einer komplexen Betrachtung. Die meisten Kinder überstehen eine verzögerte Schultergeburt und Schulterdystokie ohne Schäden, denn sowohl der Fetus als auch das Neugeborene sind gegenüber einem Sauerstoffmangel bis zu einem gewissen Ausmaß resistent. Sie haben die Fähigkeit auf ein vermindertes Sauerstoffangebot mit einer Einschränkung des Gewebestoffwechsels zu reagieren.

Mir sind viele Fallberichte bekannt, in denen die Kinder auch nach einem Zeitintervall von 5 Minuten zwischen Kopfund Schultergeburt vital zur Welt kamen. Ebenso berichten mir aber auch
Kolleginnen bei meinen Schulterdystokie-Fortbildungen von Fällen, bei denen das Kind nach nur wenigen Minuten schwer deprimiert geboren wurde. Oft kamen zur Schulterentwicklung verschiedene Maßnahmen zum Einsatz und es kann nicht ausgeschlossen werden, dass einige davon (z.B. Ziehen und Drehen am Kopf) zusätzlich das Kindswohl beeinträchtigt haben.

Wir müssen beachten, dass Kinder, die bereits im Zeitraum vor der Geburt ihres Kopfes einer verminderten Sauerstoffversorgung ausgesetzt waren, in ihren Stellreflexen beeinträchtigt sind und die Schulterrotation nur mangelhaft durch eigene Bewegungen unterstützen können. Diese Kinder werden dann mit der nächsten Wehe nicht geboren, weil ihre Schultern im hohen Gerad- oder tiefen Querstand verharren (Hildebrand nennt das sekundäre Schulterdystokie).

Deprimierte Kinder sollten darum eher zur Schulterrotation eine innere manuelle Unterstützung bekommen (6).

In jedem Fall muss davon ausgegangen werden, dass ein Kind durch Erleiden einer Schulterdystokie einem erhöhten Morbiditätsrisiko ausgesetzt ist. In Folge von Schulterdystokien gab es nach einer Studie von Sandmire und O'Halloin (9) 4,3\% schwere Azidosen, 2,9\% Mekoniumaspirationen, 7,9\% Totgeburten und 2,9\% neonatale Todesfälle. Je nach Untersuchungskollektiv wird eine perinatale Mortalität von 1,9-29\% angegeben (3).
Um dem komplexen Thema gerecht zu werden, möchte ich 3 Unterfragen bearbeiten:

1. Wann handelt es sich um eine Schulterdystokie, gibt es klare Zeitvorgaben?

2. Wird das Kind nach Geburt des Kopfes ausreichend mit Sauerstoff versorgt?

3. Wann sollen Hebammen/Geburtsmediziner mit Maßnahmen zur Beschleunigung der Schulterentwicklung beginnen?

\section{Wann handelt es sich um eine Schulterdystokie?}

In der Fachliteratur finden sich nur ganz selten konkrete Angaben über den physiologischen Zeitraum zwischen Kopf- und Schultergeburt. Ich vermute, weil die Dauer dieser Geburtsphase individuell recht verschieden ist und nie in einer größeren Studie gemessen wurde. Etliche von mir gefragte Hebammen gaben als Antwort „mit der nächsten Wehe" und konnten keine konkreten Zeitangaben machen, da sie bei normalen Geburten erst nach der Kindsentwicklung auf die Uhr sehen. Ihre subjektiven Schätzungen lagen bei 1-2 Minuten für Land- und 3-5 Minuten für Wassergeburten und decken sich mit meinen Beobachtungen.

Das längere Zeitintervall im warmen Wasser erklärt sich durch die meist niedrigere Wehenfrequenz bei Wassergeburten. Oft hat die Frau in der Durchtrittsphase (AP) nur alle 4-5 Minuten eine Wehe, und so ist auch nach der Kopfgeburt nicht eher mit der nächsten Wehe zu rechnen. 
Bezüglich der physiologischen Dauer zwischen Kopf- und Schultergeburt legen sich die meisten Fachautoren nicht durch Minutenangaben fest, sondern empfehlen auf die nächste(n) Wehe(n) zu warten.

Diese Empfehlungen zeigen, dass je nach Wehenfrequenz ein Zeitfenster von 1-3 Minuten als normal angesehen werden können.

\section{Einige Zitate aus geburtshilflichen Lehrbüichern:}

- „Da das Kind nach der Geburt des Kopfes bereits atmen kann, so eilt die Geburt der Schultern durchaus nicht so sehr. Man hüte sich vor jeder Überstürzung.“ (Jaschke, 1920).

- „Es dauert im Durchschnitt 1,5 Minuten bis nach Geburt des Kopfes die nachfolgende Wehe den Rumpf herausbefördert." (W. Stoeckel, 1948).

- „Man kann nach Geburt des Kopfes ohne das geringste Risiko auf die nächsten Wehen warten und die Schultern in Ruhe durchtreten lassen." (Rockenschaub, 2001)

- „Zwischen Kopfgeburt und Schulterentwicklung soll man abwarten: Die Geburt des Rumpfes soll mit einer der nächsten Wehen spontan vor sich gehen.“ (Dudenhausen, 2011)

In einem Lehrbuch fand ich eine Zeitvorgabe, die ein Abwarten der spontanen Schulterentwicklung kaum möglich macht:

- „Bei normalen Geburtsverläufen beträgt das Zeitintervall zwischen Geburt des Kopfes bis zur Entwicklung des Körpers maximal 60 Sekunden“ (J. Gniers u. K.T.M. Schneider, 2011).

Wann eine verzögerte Schultergeburt als Schulterdystokie klassifiziert wird, ist unter Geburtshelfern nicht klar definiert und zeigt sich an den sehr unterschiedlichen Literaturangaben zur Häufigkeit. Diese liegen z.B. bei Kindern mit einem Geburtsgewicht $<4000 \mathrm{~g}$ zwi- schen $0,15 \%$ und 1,7\% (Unterschied 11-facher Wert!), bei Kindern $>4500 \mathrm{~g}$ zwischen 4,2\% und 35,7\% (Unterschied 8 -facher Wert).

In Fachkreisen wird seit längerem darüber diskutiert, ob das heute häufiger beschriebene Phänomen der Schulterdystokie wirklich eine Folge der vermehrt übergewichtigen Kinder und Mütter ist, oder doch eher als Folge von zu frühen Manipulationen am Kopf durch ungeduldige Hebammen und Geburtshelfer zu sehen ist. Ich denke, beide Ursachen sind für Schulterdystokien verantwortlich. Für die zweite spricht, dass die vielerorts übliche aktive Schulterentwicklung durch Kopfabsenkung in Richtung Kreuzbein, sowie Drehen und Ziehen am Kopf das Kind in seinen Reflexen bei der Schulterrotation behindert und ihm zusätzlich Schmerzen und Stress beschert.

\section{Sauerstoffversorgung}

Das Kind wird nach der Geburt des Kopfes in der Regel weiter über die Nabelschnur versorgt. Die plazentare Durchblutung ist jetzt aber etwas geringer, da das Uterusvolumen und damit die Plazentahaftfläche nach der Kopfgeburt kleiner geworden sind.

Bei manchen Kindern werden leichte Atemaktivitäten beobachtet, erkennbar am leichten Stöhnen oder Greinen des geborenen Kopfes. Weil der kindliche Brustkorb jedoch vom unteren Uterinsegment und der Vagina eingeengt ist, kann er sich noch nicht komplett für einen tiefen Atemzug ausdehnen. (Die Idee einer Kollegin, bei prolongierter Schulterentwicklung das Kind mit der Maske zu beatmen, wurde meines Wissens noch nicht ausprobiert.)

Laut einer älteren Studie (12) fällt der Nabelschnur-pH-Wert in dieser Situation um etwa 0,04 Einheiten pro Minute $a b$ (3). Das bedeutet, dass ein Kind mit einem pH von 7,25 bei der Kopfgeburt nach 5 Minuten einen pH von 7,05

\section{Definitionen}

Azidamie: erhöhte Konzentration von Wasserstoffionen mit Erniedrigung des pH-Wertes im Blut

Azidose: erhöhte Konzentration von Wasserstoffionen mit Erniedrigung des pH-Wertes im Blut und im Gewebe

Hypoxämie: verminderter Sauerstoffgehalt mit erniedrigtem $\mathrm{pO}_{2}$-Wert im Blut

Hypoxie: verminderter Sauerstoffgehalt mit erniedrigtem $\mathrm{pO}_{2}$ Wert im Blut und im Gewebe Asphyxie: Kombination einer Hypoxämie mit metabolischer Azidamie und einer Hypoxie des Gewebes. Verursacht eine Atemdepression beim Neugeborenen und kann Organschädigungen hervorrufen (nach Schneider, Gniers 2011).

hätte. Dies ist eine mittelgradige Übersäuerung des Blutes, aber noch keine gravierende Asphyxie.

Als Grenzwerte für ein deutlich erhöhtes Asphyxie-Risiko sind ein Nabelschnurarterien-pH von $<7,00$ und ein Basendefizit von $<12$ mmol/l gut belegt (1).

Diese Aussage wurde auch in anderen Untersuchungen bestätigt (10).

Bezüglich der Sauerstoffversorgung in der Phase zwischen Kopf und Schultergeburt sind wir auf Vermutungen angewiesen, sie kann gut oder schlecht sein:

- Die Nabelschnur wird gut durch-

blutet, das Kind weiterhin gut versorgt, oder

- die Nabelschnurdurchblutung ist nach der Kopfgeburt durch Druck bzw. Zug herabgesetzt bis unterbrochen. 


\begin{abstract}
- Die Atmung des Kindes beginnt noch vor der Schultergeburt, oder - die Lungenatmung wird aufgrund starker Thorax-Kompression durch das untere Uterinsegment und die Vagina total behindert.
\end{abstract}

Auch das CTG liefert nach der Kopfgeburt keine sicheren Daten. Zum einen weil eine leichte Bradykardie in dieser Phase als physiologisch anzusehen ist, zum anderen weil sich die fetale Herzfrequenz jetzt nicht gut ableiten lässt. Die meisten Geburtsmediziner fordern auch keine weitere FHF-Überwachung bei einer verzögerten Schulterentwicklung, sondern lassen das CTG nur zur leichteren postpartalen Bestimmung des Zeitraums zwischen Kopf- und Rumpfgeburt weiterlaufen.

Einzig ein antepartal pathologisches FHF-Muster muss unser Handeln beschleunigen.

\section{Zeitpunkt für eine Beschleuni- gung der Schultergeburt}

Nach der Geburt des Kopfes sollte bei einem normalen Verlauf ohne Manipulation am Kopf die spontane Schulterdrehung abgewartet werden, sie erfolgt in der Regel vor oder mit der nächsten Wehe.

Wenn es dem Kind antepartal gut ging, kann auch noch auf die übernächste Wehe gewartet werden.

\section{Zeigte das Kind antepartalen Stress}

(z.B. suspektes oder pathologisches FHF-Muster), ist es günstig, die Mutter bereits nach ca. $1 / 2$ Minute zu leichten Beckenbewegungen anzuregen (Lagewechsel, Beckenschaukeln, -kreisen), um keine Zeit zu verlieren.

\section{Erscheint der Kopf aufgesetzt (turtle} syndrom) oder hat die Hebamme ein „ungutes Gefühl“ (trotz normalem CTG), empfehle ich wegen der unklaren Sauerstoffversorgung nach Kopfgeburt auch innerhalb der ersten Minute mit einer leichten mütterlichen Beckenmobilisation $\mathrm{zu}$ beginnen, da diese Maßnahme keinen Schaden anrichtet. Außerdem können mütterliche Bewegungen die nächste Wehe beschleunigen, das Kind zu Bewegungen anregen und verhindern, dass am Kopf manipuliert wird, zum Beispiel

\section{- bei Rückenlage-Geburten das}

Becken mehrmals anheben lassen (wie um die Unterlage zu wechseln) oder zur Seitenlage drehen und das obere Bein vor- und zurückbewegen (4),

- bei Vierfüßler-Geburten die Frau auf die Knie aufrichten und abwechselnd ein Bein zum Hirtenstand aufstellen lassen (5),

- bei Wassergeburten die Frau leicht zur Seite drehen und das Becken vor- und zurückkippen lassen, - bei Hockergeburten die Frau vorund zurückbeugen lassen oder aufstehen und Beckenkreisen oder in den Vierfüßlerstand bringen.

Ein langsam blau anlaufender Kopf ist kein Grund zum raschen Handeln.

Denn er ist kein Zeichen einer Hypoxie, sondern wird durch eine Abflussbehinderung im Bereich der Halsvenen verursacht - dadurch staut sich das Blut im Kopfbereich.

\section{Praxisempfehlung:}

- Nach einem Geburtsverlauf mit antepartal normalem bis suspektem CTG kann nach einer frühen leichten Beckenmobilisation eine weitere Mobilisation erfolgen (z.B. McRoberts-Manöver oder Vierfüßlerstand), bevor mit der inneren Rotation begonnen wird.

- Nach pathologischem CTG wird nach einer frühen erfolglosen Mobilisation rasch mit der inneren Rotation der Schultern begonnen (z. B. Manöver nach Rubin oder Woods).

\section{Literatur}

1 Andres RL et al. (1999): Association between umbilical bloodgas perameters and neonatal morbidity and death in neonates with pathologic fetal acidemia. Am J Obstet Gynecol. 181: 867-871

2 Dudenhausen JW: Praktische Geburtshilfe mit geburtshilflichen Operationen. 21. Aufl. de Gruyter, Berlin 2011

3 Gniers J u. Schneider KTM: Schulterdystokie. In: Schneider/Husslein/Schneider: Die Geburtshilfe. 4. Aufl. Springer, Berlin 2011

4 Harder U (2005): Sofortmaßnahmen bei Schulterdystokie. Die Hebamme 18: 138-145

5 Hildebrand S, Göbel E: Geburtshilfliche Notfälle. Hippokrates, Stuttgart 2008

6 Hildebrand S (2013) Notfallmanagement bei Schulterdystokie - erst denken, dann handeln. Die Hebamme 21: 19-23

7 Jaschke RT von, Pankow O: Lehrbuch der Geburtshilfe. Julius Springer, Berlin 1920

8 Rockenschaub A: Gebären ohne Aberglaube. Fibel und Plädoyer für die Hebammenkunst. 2. Aufl. Facultas, Wien 2001

9 Sandmire HF, O'Halloin TJ (1988) Shoulder dystocia: its incidence and associated risk factors. Int J Gynecol Obstet 26: 65-73

10 Schneider H, Gniers J: Intrapartale Asphyxie. In: Schneider/Husslein/ Schneider: Die Geburtshilfe. 4. Aufl. Springer, Berlin 2011

11 Stoeckel W: Lehrbuch der Geburtshilfe. Gustav Fischer, Jena 1948

12 Wood C et al. (1973) Time - an important variable in normal delivery. J Obstet Gynecol Br Commonw 80: 295-300

\section{Anschrift der Autorin:}

Ulrike Harder

Lehrerin für Hebammenwesen

Vivantes Klinikum Neukölln

IbBG Hebammenschule

Rudowerstr. 48, 12351 Berlin

E-Mail: ulrike.harder@gmx.de 